Messrs. Weiss have constructed for me a simple pair of strabismus forceps based on the well known Spencer Wells' artery forcepsmodifications include much lighter shanks more suitable for ophthalmic work, whilst the jaws angled on the edge at an angle of $45^{\circ}$ are capable of light but variable pressure. These jaws are flexible and grooved in a direction parallel to the muscle fibres so as to avoid crushing them.

This instrument has been in use at the Royal Eye Hospital for several months and the main advantages have been found to be in the speed of adjustment and manipulation of the muscle which is subjected to little or no trauma.

\title{
ANNOTATION
}

\section{Preventible Blinding Diseases of Childhood}

This is the title of a series of three Dr. Elizabeth Matthai lectures given in the University of Madras at the end of last year by Lt.-Col. R. E. Wright, C.I.E., and printed in the journal of Madras University. Naturally the lectures deal with such affairs as primarily affect the population of the Southern Presidency and are a useful addition to our knowledge.

The first lecture deals with Deficiency Disease and Trachoma. The deficiency disease which accounts for so much preventible blindness in children in Southern India is the avitaminosis known as keratomalacia. Wright gave his audience a good account of the signs and progress of the disease. The main deficiency in the diet concerns vitamin $\mathrm{A}$, but vitamins $\mathrm{B}, \mathrm{C}$ and $\mathrm{D}$ are possibly also concerned. Wright estimates that approximately three per thousand of his hospital cases in the year investigated, which showed partial blindness of such a degree as seriously to interfere with the earning capacity in after life, were due to keratomalacia. Treatment by crude cod-liver-oil has proved the most serviceable method in his hands, and he finds that a small dose in the form of an emulsion (for small children, $7 \frac{1}{2}$ minims) usually works well. In very marasmic infants who are too ill to take cod-liver-oil by the mouth or to tolerate it by the intestinal tract it has been found possible to save life by wrapping the child in a flannel binder soaked in cod-liver-oil.

Trachoma was linked with deficiency disease in the first lecture for convenience of spacing the subject matter. Wright gives some interesting percentages of cases under treatment for trachoma in 
various parts of India. Thus, at Lucknow 24 per cent. of cases were being treated for trachoma; at Lahore, 50 per cent.; Bombay, 14.5 per cent.; Madras Presidency, 3.5 per cent.; Burma, 75 per cent. He considers the figures for Burma greatly in excess of the true percentage. Wright condemns trachoma as "a disease of the great unwashed, who fug in frowsy overcrowded dwellings, at least these types and conditions appear to mark reservoirs of the affection."

The blinding effect of trachoma in childhood is relatively small.

The difficulties of prevention in a country like India are emphasized. Mysore would seem to be the most progressive state, and at Bangalore compulsory medical inspection of students in all grades of education is legislated for, and there are adequate arrangements for the treatment of cases in suitable centres, and for financial help to the needy and deserving for this purpose.

In India trachoma would appear to affect Mohammedans rather than Hindus; in Southern Indian Hindus it is more common in Brahmins. It appears to be commoner among Mohammedan women, and Wright has been informed by Col. Kirwan that it is relatively uncommon among Bengalis.

Wright does not refer to the fact that in Egypt and Palestine, centres with a very high percentage, it is not the trachoma per se that leads to so much blindness but trachoma + acute conjunctival infections. Does not the same fact prevail in India?

Wright's second lecture dealt chiefly with Venereal Disease.

Ophthalmia neonatorum is relatively uncommon in India and the attacks seem to be less severe than in the occident. The figures for the Government Hospital, Madras, for ten years, work out at about 2.1 per 1,000, a far lower incidence than occurs in keratomalacia.

Congenital syphilis, while fairly common in India, would seem to give rise to fewer ophthalmic complications than ophthalmia neonatorum. Small-pox is of course a frequent cause of blindness in the East. An interesting table analysing 726 corneal opacities seen in 1929 gives 59 as following small-pox; 2, ophthalmia neonatorum; 92, irritant remedies; 68 , injuries ; 54 , ulcers from acute catarrh, phlyctenular, etc., conjunctivitis ; 41 , trachoma ; 18 , keratomalacia; 14 , specific (congenital or acquired). Of 143 anterior staphylomata in the same year, 16 followed small-pox ; 35, irritant remedies; 31 , injuries; 6, keratomalacia ; 53, untraceable.

Particularly interesting are the lecturer's remarks on the results of Irritant Remedies. "The ignorance and superstition which prevails is almost unbelieveable in this connexion." A large field for prevention is here presented; and it is to be hoped that the results of education, posters, leaflets and other propaganda will lead to better things. Wright's third lecture was on Hereditary Diseases. 